\title{
Magnetic resonance quantification of non-Gaussian water diffusion in hepatic fibrosis staging: a pilot study of diffusion kurtosis imaging to identify reversible hepatic fibrosis
}

\author{
Tang Liu"^, Jiawei Hü, Yajie Liu, Honghai Chen, Dongmei Guo \\ Department of Radiology, The Second Affiliated Hospital of Dalian Medical University, Dalian, China \\ Contributions: (I) Conception and design: D Guo, J Hu; (II) Administrative support: D Guo; (III) Provision of study materials or patients: Y Liu; (IV) \\ Collection and assembly of data: T Liu, J Hu; (V) Data analysis and interpretation: Y Liu, H Chen; (VI) Manuscript writing: All authors; (VII) Final \\ approval of manuscript: All authors. \\ \#These authors contributed equally to this work. \\ Correspondence to: Dongmei Guo, MD; Honghai Chen, MD. Department of Radiology, The Second Affiliated Hospital of Dalian Medical University, \\ Dalian, China. Email: drguodong@163.com; cmuboy@163.com.
}

\begin{abstract}
Background: This study aimed to evaluate the diagnostic accuracy of diffusion kurtosis imaging (DKI) in differentiating early hepatic fibrosis (HF) from normal liver and advanced HF in rabbits.

Methods: A total of 35 healthy New Zealand white rabbits were included in the study. A model of HF was established in 30 rabbits through subcutaneous injections of $50 \%$ carbon tetrachloride $\left(\mathrm{CCl}_{4}\right) /$ olive oil, while 5 rabbits received saline injections. The gradually increased doses of $\mathrm{CCl}_{4}$ were $0.1,0.2$, and $0.3 \mathrm{~mL} / \mathrm{kg}$ in weeks 1 to 3 , weeks 4 to 6 , and weeks 7 to 10 , respectively. Two injections were given each week. Two rabbits in the experimental group died. All rabbits underwent DKI with three b values $\left(0,500\right.$, and $\left.1,000 \mathrm{~s} / \mathrm{mm}^{2}\right)$ at week $5(\mathrm{n}=8)$, week $6(\mathrm{n}=9)$, week $7(\mathrm{n}=8)$, and week $10(\mathrm{n}=8)$. Approximately 2 liver lobes per rabbit were selected for histopathology. Mean diffusivity (MD) and mean kurtosis (MK) were calculated. Discrimination capacities of DKI parameters were analyzed and compared by receiver operating characteristic (ROC) analysis.
\end{abstract}

Results: The meta-analysis of histological data in viral hepatitis (METAVIR) scoring system was used to classify liver lobes into the control group (F0, n=0), early HF group (F1-F2, n=28), and advanced HF group (F3-F4, n=28). MD and MK values were significantly different among the three groups (all $\mathrm{P}<0.05)$. MD value was negatively correlated with increased fibrosis level, while MK value was positively correlated with increased fibrosis level $(\rho=-0.540,0.614 ; \mathrm{P}<0.05)$. The area under ROC curves (AUCs) for MD and MK were 0.886 and 0.875 , respectively, for characterization of $\mathrm{F} 0$ and $\mathrm{F} 1-\mathrm{F} 2$, and 0.975 and 0.957 for $\mathrm{F} 0$ and F3-F4. AUC for MK was 0.751 for characterization of F1-F2 and F3-F4. MD performed better than MK for characterization of F0 and F1-F2 as well as F0 and F3-F4. MK showed good differentiation performance between F1-F2 and F3-F4.

Conclusions: Our results showed that DKI contributed to discriminating reversible early HF from normal liver and advanced HF and as a result, showed promise for use in HF diagnosis.

Keywords: Hepatic fibrosis (HF); staging diagnosis; magnetic resonance imaging (MRI); diffusion kurtosis imaging (DKI)

Submitted Aug 30, 2021. Accepted for publication Oct 22, 2021.

doi: $10.21037 / \mathrm{atm}-21-4884$

View this article at: https://dx.doi.org/10.21037/atm-21-4884

^ ORCID: 0000-0001-7367-5509. 


\section{Introduction}

Hepatic fibrosis (HF), a common liver disease, is caused by the excessive accumulation of extracellular matrix (ECM) proteins rich in collagen I and III. HF can progress to irreversible cirrhosis and liver failure, ultimately leading to hepatocellular carcinoma (HCC) and increasing morbidity and mortality worldwide (1-3). Recent studies have shown that the meta-analysis of histological data in viral hepatitis (METAVIR) scoring system makes a difference between reversible HF and unreversible HF. Early HF (F1-F2) is potentially reversible but once $\mathrm{HF}$ has progressed to an advanced stage (F3-F4), it is difficult to cure (2-5). Therefore, reliable methods for evaluating HF, monitoring treatment response to antifibrotic agents, and detecting the progression of $\mathrm{HF}$ are desirable $(6,7)$.

As previous studies have reported the limitations of liver biopsy, including invasiveness, sampling variability, interobserver variability, complications, and potential adverse outcomes (8-10), an increasing interest in noninvasive tools for grading HF has emerged. In recent years, magnetic resonance imaging (MRI) has been used as a noninvasive and effective examination method in the auxiliary diagnosis of HF. Conventional diffusion-weighted imaging (DWI) which assumes water molecule movements follow a Gaussian distribution, is a rapid, non-invasive imaging technique that provides valuable information at the cellular level to distinguish normal tissue from abnormal lesions, allows for assessment of HF by apparent diffusion coefficient (ADC) (11-13), and consequently can distinguish nonfibrotic liver (F0) from cirrhotic liver (F4) (14). Further, the higher the $\mathrm{b}$ value, the more valuable $\mathrm{ADC}$ is in the diagnosis of HF stage (15). However, the disadvantage of the ADC value is that it cannot accurately diagnose the duration of HF (16). Therefore, novel imaging methods for the accurate determination of HF staging are needed.

In 2005, Jensen et al. proposed the use of diffusion kurtosis imaging (DKI) for quantifying the degree of water diffusion in biological tissues based on non-Gaussian distribution (17). Kurtosis refers to the normalized and standardized fourth central moment of water displacement distribution. Higher $\mathrm{b}$ values are calculated in a modified image postprocessing procedure to obtain quantitative information regarding the magnitude and direction of nonGaussian water diffusion displacement, including mean diffusivity (MD) and mean kurtosis $(M K)(17,18)$. DKI is a dimensionless measurement that can be either positive (more sharply peaked than Gaussian) or negative. A higher kurtosis value implies a more restricted diffusion environment.
DKI is potentially capable of characterizing chronic liver diseases, pancreatic fibrosis, and renal fibrosis (19-21) and is superior for assessing gliomas, breast cancer, and rectal adenocarcinoma compared to conventional diffusion parameters (22-24). Yoshimaru et al. (25) used DKI to classify HF stage as normal and early HF, substantial stages, and advanced HF. DKI performed better than DWI in studies by Sheng et al. and Xie et al. $(26,27)$ in differentiating rats and patients with mild or substantial fibrosis from normal individuals. Corrected apparent diffusion of DKI histogram analysis showed better diagnostic performance to liver fibrosis stages compared with ADC by Sheng et al. (28) Since the application of DKI to HF research is still at the preliminary stage, the primary objective of this study was to explore the diagnostic accuracy of DKI in differentiating early HF from normal liver and advanced HF in rabbits.

We present the following article in accordance with the ARRIVE reporting checklist (available at https://dx.doi. org/10.21037/atm-21-4884).

\section{Methods}

\section{Animal models}

Experiments were performed under a project license (No. AEE18069) granted by institutional board of Dalian Medical University, in compliance with Dalian Medical University institutional guidelines for the care and use of animals. A protocol was prepared before the study without registration. Thirty-five healthy, 6-to-8-month-old, male New Zealand white rabbits weighing $2.5-3.0 \mathrm{~kg}$ were provided by the Medical Experimental Animal Center of Dalian Medical University. Thirty rabbits were randomly allocated to the experimental group and 5 rabbits to the control group. A rabbit model of HF was established in the experimental group through subcutaneous injection of $50 \%$ carbon tetrachloride $\left(\mathrm{CCl}_{4}\right) /$ olive oil. The gradually increased doses of $\mathrm{CCl}_{4}$ were $0.1,0.2$, and $0.3 \mathrm{~mL} / \mathrm{kg}$ in weeks 1 to 3 , weeks 4 to 6 , and weeks 7 to 10 , respectively. Two injections were given each week. The control group rabbits were injected with saline for 10 consecutive weeks (Figure 1). All injections were carried out between 10:00 AM and 11:00 AM at weekends. During the modeling process, the rabbits' appetite, activity, color and depilation were closely observed.

\section{MRI}

MRI was performed at 5, 6, 7, and 10 weeks. The rabbits fasted for 8 hours and were then anesthetized with an 


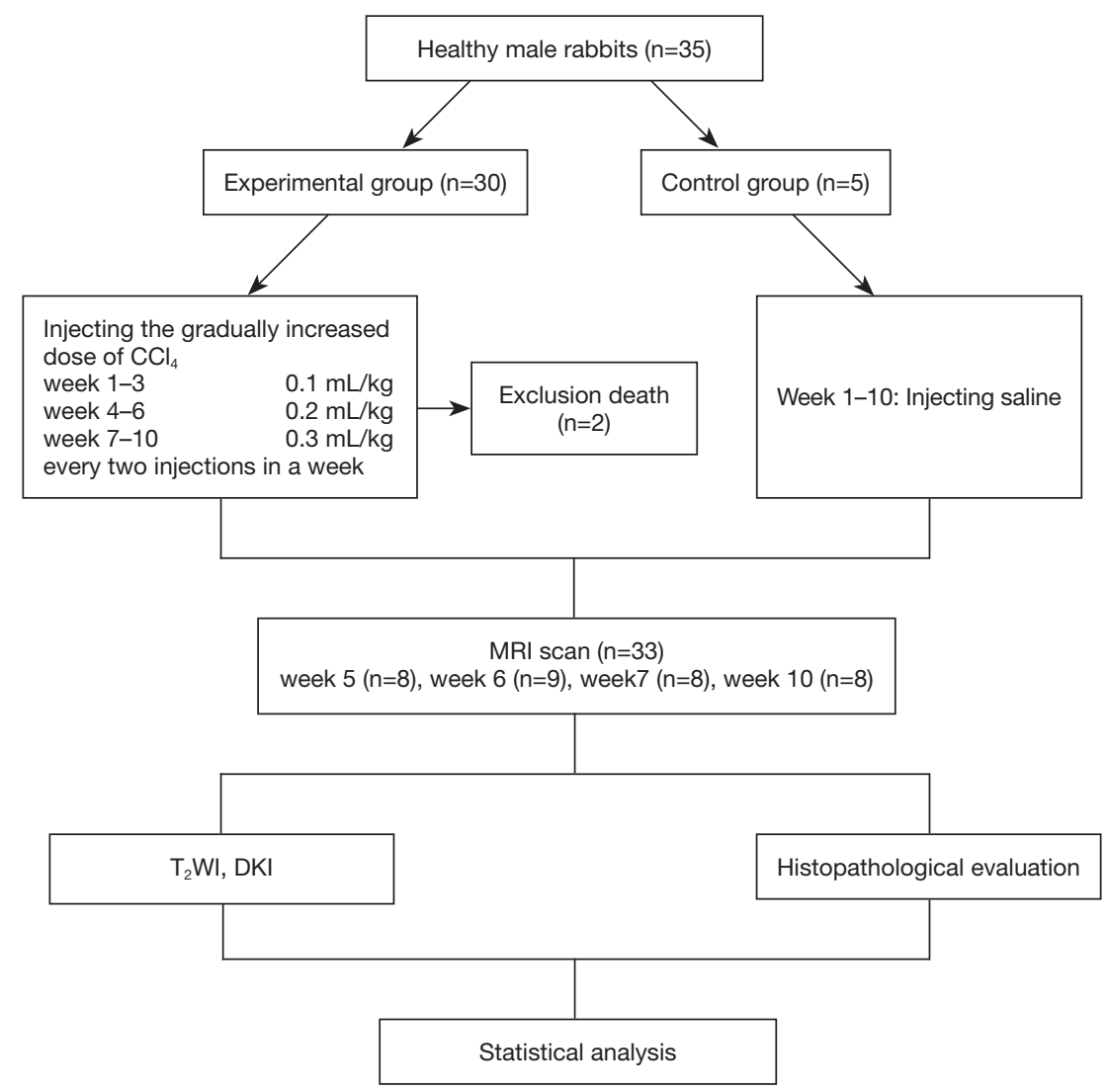

Figure 1 The experiment flow chart. Thirty healthy, 6-to-8-month-old, male New Zealand white rabbits were randomly selected for the experimental group and 5 rabbits for the control group. A rabbit model of HF was established in the experimental group through subcutaneous injections of $50 \% \mathrm{CCl}_{4} /$ olive oil. The gradually increased doses of $\mathrm{CCl}_{4}$ were $0.1,0.2$, and $0.3 \mathrm{~mL} / \mathrm{kg}$ in weeks 1 to 3 , weeks 4 to 6 , and weeks 7 to 10 , respectively. Two injections were given each week. The control group rabbits were injected with saline for 10 consecutive weeks. A total of 33 rabbits were included in the experiment; 2 rabbits in the experimental group died (1 from hepatic failure and 1 from the anesthesia). All rabbits underwent DKI with three b values $\left(0,500\right.$, and 1,000 s/mm $\left.{ }^{2}\right)$ at 5 , 6, 7, and 10 weeks. HF, hepatic fibrosis; $\mathrm{CCl}_{4}$, carbon tetrachloride; DKI, diffusion kurtosis imaging; MRI, magnetic resonance imaging; $\mathrm{T}_{2} \mathrm{WI}$, T2-weighted images.

auricular vein injection of $1.5 \mathrm{~mL} / \mathrm{kg} 10 \%$ chloral hydrate and fixed in the supine position for MRI examination. An abdominal belt was used to reduce the respiratory artifacts. The whole liver was scanned using a 3.0-T MRI scanner (Discovery MR750w, GE Healthcare, Waukesha, WI, USA) with an 8-channel phased-array knee coil for signal reception and a body coil for transmission. The routine MR examination protocol contained axial $\mathrm{T} 2$-weighted images $\left(\mathrm{T}_{2} \mathrm{WI}\right)$ according to the following scanning parameters: repetition time $(\mathrm{TR}) / \mathrm{echo}$ time $(\mathrm{TE})=2,000 / 50 \mathrm{~ms}$, slice thickness $=4 \mathrm{~mm}$, slice gap $=1 \mathrm{~mm}$, field of view (FOV) $=20 \times 20 \mathrm{~cm}$, matrix $=128 \times 128$, number of excitations $(\mathrm{NEX})$ $=4$, and time $=1$ minute 40 seconds. DKI involved spin-echo echo-planar imaging and included three $\mathrm{b}$ values $(0,500$, and $1,000 \mathrm{~s} / \mathrm{mm}^{2}$ ) and 15 diffusion directions according to the following scanning parameters: TR/TE $=4,000 / 90 \mathrm{~ms}$, slice thickness $=4 \mathrm{~mm}$, slice gap $=1 \mathrm{~mm}, \mathrm{FOV}=20 \times 20 \mathrm{~cm}$, matrix $=128 \times 128, \mathrm{NEX}=4$, flip angle $=90^{\circ}$, and time $=9$ minute 24 seconds.

\section{Imaging analysis}

All images were transferred to a workstation (Advantage Workstation 4.6, GE Healthcare) and postprocessed with vendor-supplied software by two independently trained radiologists with 3 years of MRI experience who were blinded to the histopathologic results. DKI images were used to compute MD and MK. All DKI parameters were measured for $T_{2} W I$ images. Three regions of interest (ROIs) with a mean area of $20 \mathrm{~mm}^{2}$ were placed on each 

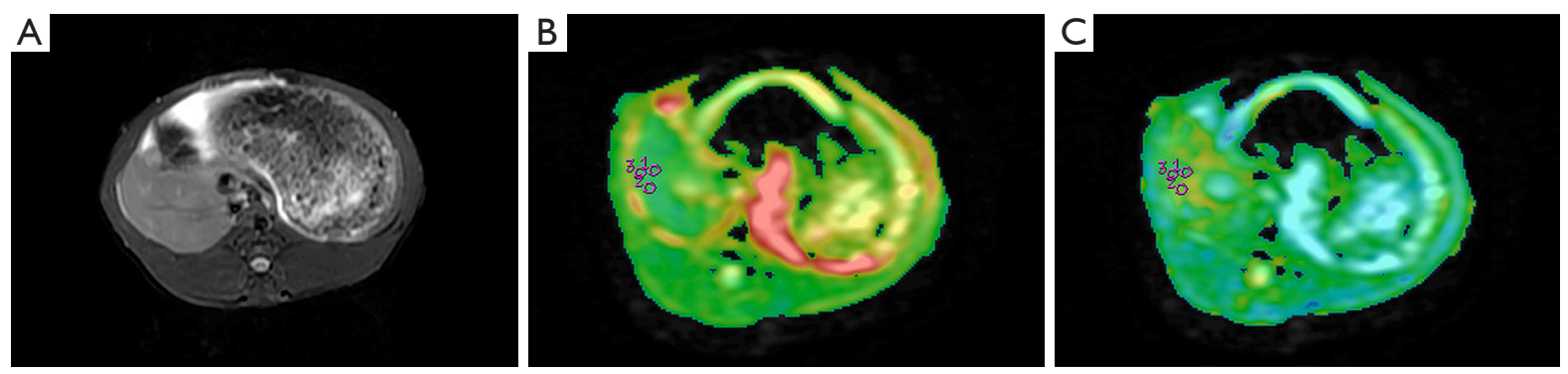

Figure $2 \mathrm{~T}_{2} \mathrm{WI}, \mathrm{MD}$, and MK maps of HF at stage F3. Three ROIs were placed on the middle lobe of the right liver. (A) $\mathrm{T}_{2} \mathrm{WI}$. (B) ROIs ranging from $20 \mathrm{~mm}^{2}$ were placed on the MD image. (C) ROIs were copied to the MK image. $\mathrm{T}_{2} \mathrm{WI}$, T2-weighted images; $\mathrm{MD}$, mean diffusivity; MK, mean kurtosis; HF, hepatic fibrosis; ROIs, regions of interest.

lobe according to its volume, and the values were recorded. The mean value of the 3 ROIs was computed as the corresponding average value of MD and MK for each liver lobe. Regions with severe artifacts, blood vessels, and the bile duct were avoided when drawing ROIs. Examples of MD and MK maps of HF are shown in Figure 2.

\section{Histopathologic examination}

After satisfactory MRI was obtained, the rabbits were killed via cervical dislocation. The livers were then removed and fixed in $10 \%$ formaldehyde. The tissue specimens were processed by a pathologist with 10 years of experience using the following steps: sampling, fixing, mending, flushing, dehydrating, hyalinizing, waxing, paraffin embedding, paraffin sectioning, hematoxylin-eosin (HE) staining, Masson 3-color staining, and mounting. The stained slices were then observed under optical microscope and assessed for HF grading in accordance with METAVIR scoring system (4): F0 = no fibrosis, F1 = mild fibrosis (portal fibrosis without septa), F2 = substantial fibrosis (periportal fibrosis and few septa), F3 = advanced fibrosis, (septal fibrosis without cirrhosis), F4 = widespread fibrosis with cirrhosis. Based on the pathological results, the liver lobes were divided into the control group (F0), early HF group (F1-F2), and advanced HF group (F3-F4). The pathological samples were selected based on the magnetic resonance images and liver lobes with artifacts were excluded. Pathological sections of liver tissues with different HF stages are shown in Figure 3.

\section{Statistical analysis}

Statistical analyses were performed using SPSS software for Windows, version 21.0 (IBM, Chicago, IL, USA). Interobserver reproducibility of MD and MK measurements was assessed using an intraclass correlation coefficient (ICC), which defined reproducibility as poor $(<0.04)$, fair to moderate $(0.4-0.75)$, and good $(>0.75)$. Data were analyzed by one-way analysis of variance (ANOVA). Post hoc tests [Fisher's least significant difference (LSD), Bonferroni, and Games-Howell] were conducted after the Levene variance homogeneity test and Kolmogorov-Smirnov normality test were performed to assess variance and normality for each group. In addition, the nonparametric Chi-square test was used as a supplementary validation for heterogeneous variance. Spearman rank correlation analysis was used to investigate the correlation between DKI parameters and HF stages (26). The area under receiver operating characteristic (ROC) curve (AUC) for each DKI parameter was computed to evaluate discrimination performance. The maximum value of the Youden index was selected as a cut-off and its sensitivity and specificity for diagnosis were also calculated. $\mathrm{P}$ value $<0.05$ was considered statistically significant.

\section{Results}

\section{Animal models and histopathologic findings}

Two rabbits in the experimental group died before MR examination; 1 as a result of hepatic failure and 1 from the anesthesia. In total, 5 control group rabbits and 28 rabbits from the experimental group were included in the analysis. Approximately 2 liver lobes per rabbit were selected for histopathology. Liver lobes were allocated to the control group (F0, $\mathrm{n}=10)$, early HF group $(\mathrm{F} 1-\mathrm{F} 2, \mathrm{n}=28)$, and advanced $\mathrm{HF}$ group (F3-F4, n=28), based on the METAVIR scoring system. 

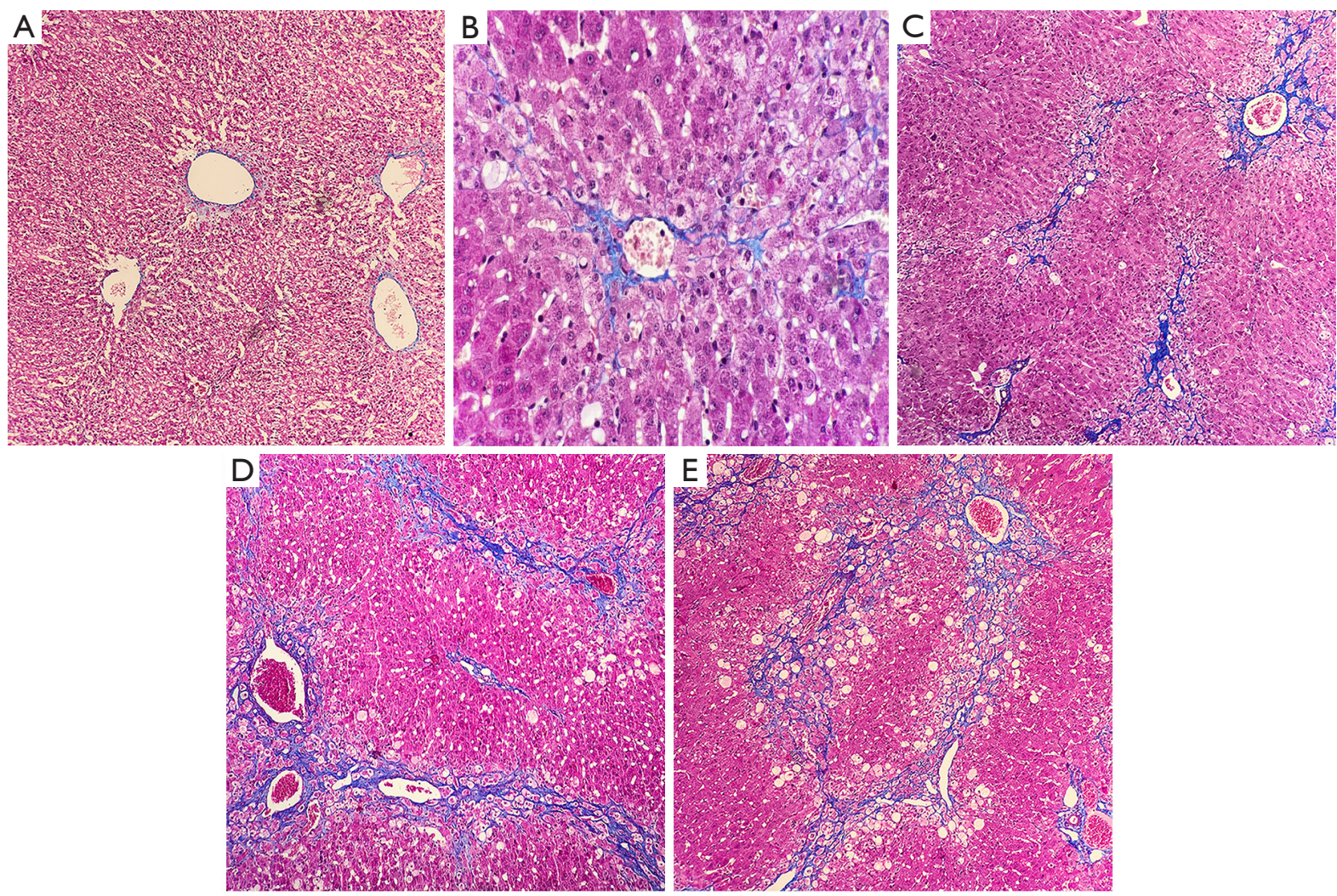

Figure 3 Masson staining (original magnification $\times 100$ ) based on the METAVIR scoring system. (A) F0, the normal control. (B) F1, mild fibrosis (portal fibrosis without septa). (C) F2, substantial fibrosis (periportal fibrosis and few septa). (D) F3, advanced fibrosis (septal fibrosis without cirrhosis). (E) F4, widespread fibrosis with cirrhosis. METAVIR, meta-analysis of histological data in viral hepatitis.

Table 1 The DKI parameters in the control group, early HF group, and advanced HF group

\begin{tabular}{lcc}
\hline Group & $\mathrm{MD}\left(\times 10^{-3} \mathrm{~mm}^{2} / \mathrm{s}\right)$ & $M K$ \\
\hline F0 $(\mathrm{n}=10)$ & $3.288 \pm 0.746$ & $0.798 \pm 0.232$ \\
F1-F2 $(\mathrm{n}=28)$ & $1.972 \pm 0.718$ & $1.168 \pm 0.372$ \\
F3-F4 $(\mathrm{n}=28)$ & $1.575 \pm 0.572$ & $1.439 \pm 0.214$ \\
\hline
\end{tabular}

$\mathrm{DKI}$, diffusion kurtosis imaging; HF, hepatic fibrosis; MD, mean diffusivity; MK, mean kurtosis.

\section{DKI parameter correlation in control group and rabbits with early and advanced $\mathrm{HF}$}

The interobserver reproducibility for the MD and MK values were good, with an ICC of 0.995 [95\% confidence interval (CI): 0.992-0.997] and 0.890 (95\% CI: $0.992-$ 0.997), respectively. The average values of MD and MK in the F0, F1-F2, and F3-F4 groups are listed in Table 1. The variance of $M D$ values was homogeneous $(\mathrm{P}=0.153)$. The LSD post hoc test showed significant differences in MD values between groups (all $\mathrm{P}<0.05$ ), while the Bonferroni post hoc test showed significant differences between $\mathrm{F} 0$ and $\mathrm{F} 1-\mathrm{F} 2$ as well as $\mathrm{F} 3-\mathrm{F} 4$ (all $\mathrm{P}<0.05$ ) but not between $\mathrm{F} 1-$ $\mathrm{F} 2$ and $\mathrm{F} 3-\mathrm{F} 4(\mathrm{P}=0.089)$. The variance of $\mathrm{MK}$ values was heterogeneous $(\mathrm{P}=0.004)$, and significant differences in $\mathrm{MK}$ values were shown between groups with the Games-Howell post hoc test $($ all $\mathrm{P}<0.05)$. In addition, the results of the Chi-square test showed that $\mathrm{MK}$ values in the advanced $\mathrm{HF}$ group were higher than in the early HF group as well as the normal control group $(\mathrm{P}=0.010)$.

\section{ROC analysis of DKI parameters for HF staging}

$M D$ and MK values were significantly correlated with the extent of HF staging (Spearman's rank correlation coefficient, $\rho=-0.540,0.614, \mathrm{P}<0.05)$. MD values were better able to identify F0 and F1-F2, and F0 and F3-F4, 
with AUCs of 0.886 (95\% CI: 0.776-0.996) and 0.975 (95\% CI: $0.975-1.000)$, compared to MK values, with AUCs of 0.875 (95\% CI: 0.730-1.000) and 0.957 (95\% CI: 0.874-1.000). MK values were better able to identify F1-F2 and F3-F4, with an AUC of 0.751 (95\% CI: 0.610-0.891), compared to MD values, with an AUC of 0.663 (95\% CI: 0.520-0.807) (Tables 2,3 and Figure 4).

\section{Discussion}

DKI is a straightforward extension of conventional DWI, with higher order diffusion to quantify the non-Gaussian water diffusion displacement in the diagnosis of HF. In the application of DKI, the higher b value can reflect the local water molecule diffusion in non-Gaussian distribution. However, because the signal-to-noise ratio (SNR) decreases as the $b$ value increases, the $b$ value used in the kurtosis model should be smaller than $3 / \mathrm{MD} \times \mathrm{MK}$. Jensen et al. (17) reported that kurtosis analysis could be carried out with lower b values. In currently published liver DKI studies, the maximum b value is $2,000 \mathrm{~s} / \mathrm{mm}^{2}(25)$. We chose three $\mathrm{b}$ values $\left(0,500\right.$, and $\left.1,000 \mathrm{~s} / \mathrm{mm}^{2}\right)$ and 15

Table 2 Comparisons of DKI parameters between groups by ANOVA and corresponding post-hoc corrections

\begin{tabular}{lcccc}
\hline \multirow{2}{*}{ Group } & \multicolumn{2}{c}{ MD } & & MK \\
\cline { 2 - 3 } \cline { 5 - 5 } & LSD & Bonferroni & & Games-Howell \\
\hline F0 vs. F1-F2 & $0.000^{*}$ & $0.000^{*}$ & & $0.003^{\star}$ \\
F0 vs. F3-F4 & $0.000^{\star}$ & $0.000^{\star}$ & & $0.000^{\star}$ \\
F1-F2 vs. F3-F4 & $0.028^{\star}$ & 0.089 & & $0.005^{\star}$ \\
\hline
\end{tabular}

*, $\mathrm{P}<0.05$. DKI, diffusion kurtosis imaging; ANOVA, analysis of variance; MD, mean diffusivity; MK, mean kurtosis; LSD, least significant difference. diffusion directions for 2 nonzero values in this experiment to achieve good images and obtain kurtosis tensor-derived parameters $(29,30)$.

\section{DKI parameter correlations in $\mathrm{HF}$}

MD and MK values were the most representative DKI parameters reflecting macroscopically and quantitatively microscopic pathological changes in liver fibrosis. Our results showed that MD values decreased as the fibrosis advanced and showed good differentiation ability between F0 and F1-F2 as well as F3-F4, which was consistent with the pathological basis of HF progression. Collagen molecules, glycosaminoglycans, and proteoglycans are deposited in the hepatic extracellular (30), leading to shrinkage of extracellular space and restricting the diffusion of water molecules. MD refers to the average movement of water in 3 main directions. Therefore, MD can more precisely reflect the water molecule diffusion movement in extracellular spaces as the extracellular space changes.

Our study also demonstrated that MK values increased as the fibrosis advanced and showed good differentiation ability between F0 and F1-F2 as well as F3-F4. MK showed good differentiation performance between F1-F2 and F3-F4. In theory, MK mainly reflects the complexity of organizational structures. When liver fibrosis continues to progress, it could be accompanied by hepatocyte swelling and inflammatory cell infiltration $(31,32)$, which would increase the microstructural complexity of cells in the liver.

\section{Diagnostic performance of DKI parameters for HF staging}

In our study, as HF progressed, MD values decreased while MK values increased, both of which were significantly

Table 3 The cut-off, AUC, sensitivity, and specificity of DKI parameters between the control group, early HF group, and advanced HF group

\begin{tabular}{lcccccc}
\hline Parameters & Group & AUC $(95 \%$ Cl $)$ & P & Cut-off & Sensitivity & Specificity \\
\hline MD & F0 vs. F1-F2 & $0.886(0.776-0.996)$ & 0.000 & 0.629 & 0.700 & 0.929 \\
& F0 vs. F3-F4 & $0.975(0.925-1.000)$ & 0.000 & 0.864 & 0.900 & 0.964 \\
& F1-F2 vs. F3-F4 & $0.663(0.520-0.807)$ & 0.036 & 0.321 & 0.357 & 0.964 \\
MK & F0 vs. F1-F2 & $0.875(0.730-1.000)$ & 0.001 & 0.664 & 0.964 & 0.700 \\
& F0 vs. F3-F4 & $0.957(0.874-1.000)$ & 0.000 & 0.864 & 0.964 & 0.900 \\
& F1-F2 vs. F3-F4 & $0.751(0.610-0.891)$ & 0.001 & 0.571 & 0.964 \\
\hline
\end{tabular}

AUC, area under receiver operating characteristic curve; DKI, diffusion kurtosis imaging; HF, hepatic fibrosis; Cl, confidence interval; MD, mean diffusivity; MK, mean kurtosis. 

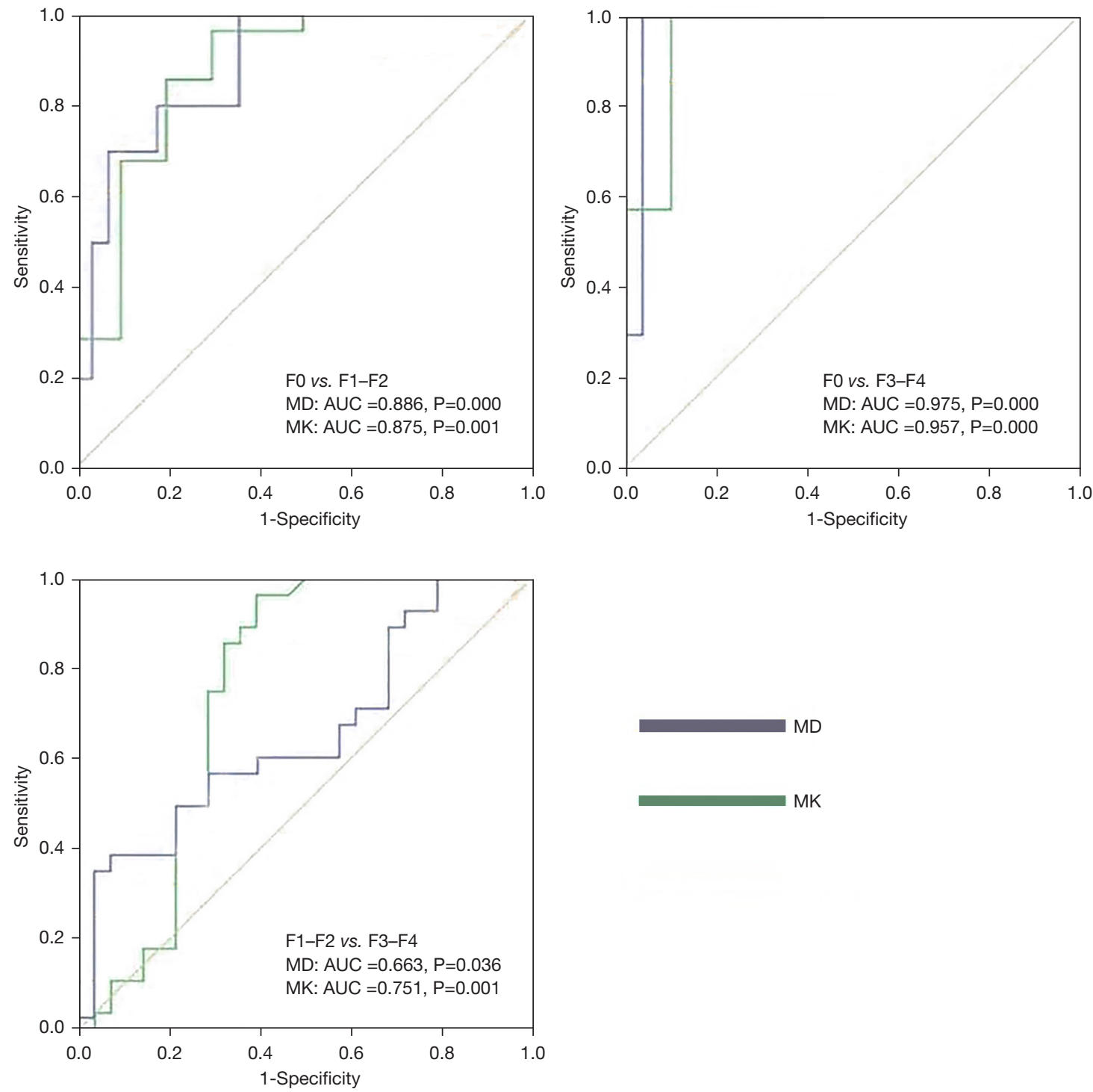

Figure 4 The ROC of DKI parameters (MD, MK) in discriminating F0 vs. F1-F2, F0 vs. F3-F4, and F1-F2 vs. F3-F4. ROC, receiver operating characteristic; DKI, diffusion kurtosis imaging; MD, mean diffusivity; MK, mean kurtosis; AUC, area under ROC curve.

correlated with the progress of $\mathrm{HF}(\mathrm{P}<0.05)$. MD and MK were promising parameters in distinguishing reversible HF from other HF stages and contributed to good differentiation diagnosis in intergroups F0, F1-F2, and F3F4. MD values had the highest diagnostic efficacy (AUC $=0.975$ and $0.886, \mathrm{P}<0.05$, respectively) in differentiating $\mathrm{F} 0$ from F3-F4 and F1-F2. Studies have shown that synthesis of ECM increased in HF while degradation decreased. Our results indicated that the reduced extracellular space and the restricted water diffusivity in the extracellular space were the main distinguishing features between normal liver and HF, which was consistent with the findings of a human study, even though the effect of overlapping HF was ignored (25).

With respect to discrimination between $\mathrm{F} 1-\mathrm{F} 2$ and $\mathrm{F} 3-\mathrm{F} 4$, the two different post hoc approaches for multicomparison corrections gave different results. The Bonferroni post hoc test, stricter than the other test, showed that $\mathrm{MK}$ values had higher diagnostic performance (AUC $=0.751, \mathrm{P}<0.05)$, implying that the complexity of hepatocyte microstructure increased significantly in advanced HF. Our results were consistent with $\mathrm{Li}$ et al. (33), while they 
differed with the findings of Sheng et al., which suggested that MK values had a weak correlation with HF compared to MD values, with low identification ability in mild and severe fibrosis and in noncirrhosis and cirrhosis (26). This might have been due to the selection of different $b$ values and because the HF models in our study avoided HF stage overlapping (25).

\section{Limitations of this study}

The present study had some limitations. First, the sample size was relatively small. Hence, detailed analysis from F0 to F4, particularly differentiation between F2 and F3, requires further study. Second, the established HF rabbit model induced by $\mathrm{CCl}_{4}$ was not exactly the same as clinical HF. Last but not the least, the effects of inflammation and steatosis of HF based on DKI parameters were not studied due to the very mild symptoms in our established models.

\section{Conclusions}

As a noninvasive functional MRI technique, DKI parameters could reflect pathophysiological and microstructural changes during HF, contributing to identification of reversible HF, and is worthy further study.

\section{Acknowledgments}

We thank Dr. Lizhi Xie and Weiyin Vivian Liu from GE Healthcare for their help in solving technical problems.

Funding: This study was supported by the Natural Science Foundation of Liaoning Province, China (No. 201602228).

\section{Footnote}

Reporting Checklist: The authors have completed the ARRIVE reporting checklist. Available at https://dx.doi. org/10.21037/atm-21-4884

Data Sharing Statement: Available at https://dx.doi. org/10.21037/atm-21-4884

Conflicts of Interest: All authors have completed the ICMJE uniform disclosure form (available at https://dx.doi. org/10.21037/atm-21-4884). All authors reported that this study was supported by the Natural Science Foundation of Liaoning Province, China (No. 201602228). The authors have no other conflicts of interest to declare.
Ethical Statement: The authors are accountable for all aspects of the work in ensuring that questions related to the accuracy or integrity of any part of the work are appropriately investigated and resolved. Experiments were performed under a project license (No. AEE18069) granted by institutional board of Dalian Medical University, in compliance with Dalian Medical University institutional guidelines for the care and use of animals. A protocol was prepared before the study without registration.

Open Access Statement: This is an Open Access article distributed in accordance with the Creative Commons Attribution-NonCommercial-NoDerivs 4.0 International License (CC BY-NC-ND 4.0), which permits the noncommercial replication and distribution of the article with the strict proviso that no changes or edits are made and the original work is properly cited (including links to both the formal publication through the relevant DOI and the license). See: https://creativecommons.org/licenses/by-nc-nd/4.0/.

\section{References}

1. Schuppan D, Afdhal NH. Liver cirrhosis. Lancet 2008;371:838-51.

2. Bataller R, Brenner DA. Liver fibrosis. J Clin Invest 2005;115:209-18.

3. Hernandez-Gea V, Friedman SL. Pathogenesis of liver fibrosis. Annu Rev Pathol 2011;6:425-56.

4. Bedossa P, Poynard T. An algorithm for the grading of activity in chronic hepatitis C. The METAVIR Cooperative Study Group. Hepatology 1996;24:289-93.

5. Lee YA, Wallace MC, Friedman SL. Pathobiology of liver fibrosis: a translational success story. Gut 2015;64:830-41.

6. Serov VV, Severgina LO. Morphologic criteria for the assessment of etiology, degree of activity and stage of the process in viral chronic hepatitis B and C. Arkh Patol 1996;58:61-4.

7. Ghany MG, Strader DB, Thomas DL, et al. Diagnosis, management, and treatment of hepatitis $\mathrm{C}$ : an update. Hepatology 2009;49:1335-74.

8. Regev A, Berho M, Jeffers LJ, et al. Sampling error and intraobserver variation in liver biopsy in patients with chronic HCV infection. Am J Gastroenterol 2002;97:2614-8.

9. Bedossa P, Dargère D, Paradis V. Sampling variability of liver fibrosis in chronic hepatitis C. Hepatology 2003;38:1449-57.

10. Rockey DC, Caldwell SH, Goodman ZD, et al. Liver 
biopsy. Hepatology 2009;49:1017-44.

11. Faria SC, Ganesan K, Mwangi I, et al. MR imaging of liver fibrosis: current state of the art. Radiographics 2009;29:1615-35.

12. Wang QB, Zhu H, Liu HL, et al. Performance of magnetic resonance elastography and diffusion-weighted imaging for the staging of hepatic fibrosis: a meta-analysis. Hepatology 2012;56:239-47.

13. Koinuma M, Ohashi I, Hanafusa K, et al. Apparent diffusion coefficient measurements with diffusionweighted magnetic resonance imaging for evaluation of hepatic fibrosis. J Magn Reson Imaging 2005;22:80-5.

14. Sandrasegaran K, Akisik FM, Lin C, et al. Value of diffusion-weighted MRI for assessing liver fibrosis and cirrhosis. AJR Am J Roentgenol 2009;193:1556-60.

15. Li H, Chen TW, Chen XL, et al. Magnetic resonancebased total liver volume and magnetic resonance-diffusion weighted imaging for staging liver fibrosis in mini-pigs. World J Gastroenterol 2012;18:7225-33.

16. Cheung JS, Fan SJ, Gao DS, et al. Diffusion tensor imaging of liver fibrosis in an experimental model. J Magn Reson Imaging 2010;32:1141-8.

17. Jensen JH, Helpern JA, Ramani A, et al. Diffusional kurtosis imaging: the quantification of non-gaussian water diffusion by means of magnetic resonance imaging. Magn Reson Med 2005;53:1432-40.

18. Lu H, Jensen JH, Ramani A, et al. Three-dimensional characterization of non-gaussian water diffusion in humans using diffusion kurtosis imaging. NMR Biomed 2006;19:236-47.

19. Anderson SW, Barry B, Soto J, et al. Characterizing nongaussian, high b-value diffusion in liver fibrosis: Stretched exponential and diffusional kurtosis modeling. J Magn Reson Imaging 2014;39:827-34.

20. Kjølby BF, Khan AR, Chuhutin A, et al. Fast diffusion kurtosis imaging of fibrotic mouse kidneys. NMR Biomed 2016;29:1709-19.

21. Zhang T, Lu Y, Yang B, et al. Diffusion metrics for staging pancreatic fibrosis and correlating with epithelialmesenchymal transition markers in a chronic pancreatitis rat model at 11.7T MRI. J Magn Reson Imaging 2020;52:197-206.

22. Zhao J, Wang YL, Li XB, et al. Comparative analysis of the diffusion kurtosis imaging and diffusion tensor imaging in grading gliomas, predicting tumour cell proliferation and IDH-1 gene mutation status. J Neurooncol 2019;141:195-203.

23. Sun K, Chen X, Chai W, et al. Breast cancer: diffusion kurtosis $\mathrm{mr}$ imaging-diagnostic accuracy and correlation with clinical-pathologic factors. Radiology 2015;277:46-55.

24. Zhu L, Pan Z, Ma Q, et al. Diffusion kurtosis imaging study of rectal adenocarcinoma associated with histopathologic prognostic factors: preliminary findings. Radiology 2017;284:66-76.

25. Yoshimaru D, Miyati T, Suzuki Y, et al. Diffusion kurtosis imaging with the breath-hold technique for staging hepatic fibrosis: a preliminary study. Magn Reson Imaging 2018;47:33-8.

26. Sheng RF, Wang HQ, Yang L, et al. Diffusion kurtosis imaging and diffusion-weighted imaging in assessment of liver fibrosis stage and necroinflammatory activity. Abdom Radiol (NY) 2017;42:1176-82.

27. Xie S, Li Q, Cheng Y, et al. Differentiating mild and substantial hepatic fibrosis from healthy controls: a comparison of diffusion kurtosis imaging and conventional diffusion-weighted imaging. Acta Radiol 2020;61:1012-20.

28. Sheng RF, Jin KP, Yang L, et al. Histogram analysis of diffusion kurtosis magnetic resonance imaging for diagnosis of hepatic fibrosis. Korean J Radiol 2018;19:916-22.

29. Jensen JH, Helpern JA. MRI quantification of nonGaussian water diffusion by kurtosis analysis. NMR Biomed 2010;23:698-710.

30. Huang Y, Chen X, Zhang Z, et al. MRI quantification of non-Gaussian water diffusion in normal human kidney: a diffusional kurtosis imaging study. NMR Biomed 2015;28:154-61.

31. Neubauer K, Saile B, Ramadori G. Liver fibrosis and altered matrix synthesis. Can J Gastroenterol 2001;15:187-93.

32. Hu G, Liang $W, W u ~ M$, et al. Staging of rat liver fibrosis using monoexponential, stretched exponential and diffusion kurtosis models with diffusion weighted imagingmagnetic resonance. Oncotarget 2018;9:2357-66.

33. Li J, Wang D, Chen TW, et al. Magnetic resonance diffusion kurtosis imaging for evaluating stage of liver fibrosis in a rabbit model. Acad Radiol 2019;26:e90-7.

(English Language Editor: A. Muijlwijk)

Cite this article as: Liu T, Hu J, Liu Y, Chen H, Guo D. Magnetic resonance quantification of non-Gaussian water diffusion in hepatic fibrosis staging: a pilot study of diffusion kurtosis imaging to identify reversible hepatic fibrosis. Ann Transl Med 2021;9(20):1569. doi: 10.21037/atm-21-4884 